\title{
Typlandschaften der Schweiz - methodische, didaktische und schulpraktische Überlegungen
}

\section{Typlandschaften im Unterricht}

\subsection{Landschaftstypen und Typlandschaften}

Thematische Problemstellungen mit Hilfe geeigneter Regionalbeispiele erarbeiten: Diese Aufgabe des heutigen Geographieunterrichtes führt zur Suche entsprechender Landschaften. Jeder Versuch, mit Typlandschaften zu arbeiten, beginnt damit, die landschaftliche Vielfalt der Erdoberfläche in überschaubaren Kategorien zusammenzufassen. Gefragt sind dabei entscheidende Kriterien, die ausgefiltert und zusammengefaßt werden können: «Typlandschaften unterscheiden sich in der differenzierten Ausprägung gleicher Merkmale» (AERNI 1993). JEANNERET (1993) untersuchte und verglich sprachübergreifend den Umgang mit dem Begriff «Typlandschaft» und schlägt die Definition «Die Typlandschaft ist ein Vertreter oder ein Beispiel eines Landschaftstypes» vor. AERNI (1993) zeigte denn auch am Beispiel der Schweiz drei verschiedene Typologisierungen aufgrund unterschiedlicher Zielsetzungen.

Allen Typologisierungen ist dabei das Vorgehen gemeinsam: Originale Landschaften werden verglichen, Ähnliches zusammengefaßt und hinsichtlich der Aussagekraft beurteilt (vgl. Abb. 1). Gemeinsame Aussagen, die sich verallgemeinern lassen, bilden die Grundlage der Landschaftstypisierung. Landschaften, aus denen sich eine große Vielfalt von Aussagen zur Verallgemeinerung ableiten lassen, werden als Typlandschaften ausgezeichnet. Eine Landschaft, die entscheidende Elemente einer Faltenjuralandschaft aufweist (Klus, Karstphänomene, synklinal ausgerichtete Siedlungen und Hauptverkehrsachsen, quer zum Tal gerichtete Nutzungsstufen) wie z. B. das Val de Travers (ARBEITSBLATT Nr. 3), darf daher in eine solche Liste aufgenommen werden.

Allgemein gültige und unterrichtsrelevante Aussagen gewinnt der Betrachter durch die Analyse der Landschaftselemente und der raumprägenden Prozesse. Drei Beispiele:

1. In einem Karstgebiet ist das Oberflächenabflußnetz nur rudimentär ausgebildet (ARBEITSBLATT Nr. 3, Val de Travers).

2. Die Entsumpfung und Korrektur der Talböden im 19. Jahrhundert schuf die Voraussetzungen zur modernen Verkehrserschließung, zur Intensivierung der Landwirtschaft und zur Siedlungserweiterung in die flachen Talböden (ARBEITSBLATT Nr. 14, Zihlebene bei Cressier).
3. Die traditionelle alpine Kulturlandschaft orientierte sich an der sorgfältigen Nutzung der Höhenstufen quer zum Talverlauf. Die heutige einseitige Bewertung der Alpen als Erholungsraum und Verkehrshindernis reduziert die Nutzung des alpinen Raumes auf Talachsen und die hochalpinen Gebiete (Wintersport), während die Hanglagen (Maiensäße) langsam verganden (ARBEITSBLATT Nr. 10, Lötschental).

Ob eine Landschaft als Typlandschaft im Unterricht eingesetzt wird, hängt vom entsprechenden Lernziel, von der leichten Erfaßbarkeit der entscheidenden Landschaftselemente und der landschaftsbildenden bzw. -verändernden Prozesse ab. Aber auch die räumliche Nähe und die Möglichkeit einer Bearbeitung vor Ort (Exkursion, Studienwoche) sind oftmals entscheidende Auswahlkriterien.

\subsection{Landschaftsdarstellungen im Geographieunterricht zwischen "typisch" und "original"}

In der Geographie, einem Schulfach, das mit einem klar enzyklopädischen Bildungsauftrag im 19. Jahrhundert in den Maturitätsfächerkatalog aufgenommen wurde (die Weite, Vielfalt und Größe der Welt sollte gezeigt werden), läßt sich heute eine allumfassende Behandlung stofflich nicht mehr bewältigen und didaktisch nicht mehr begründen. Über Typlandschaften wird nun ein Weg zu einem exemplarischen Vorgehen eröffnet.

Es ist bereits dargelegt worden, daß mit Typlandschaften auch ein nachhaltiger Beitrag zu einem verantwortungsbewußten Umgang mit der Umwelt geleistet werden kann (HASLER 1993). So leisten Typlandschaften vor allem wichtige Beiträge im kognitiven und arbeitsmethodischen Bereich (Abb. 2).

Gleichzeitig muß sich die Geographielehrkraft aber auch der Grenzen der Typlandschaften bewußt werden. Typlandschaften erzeugen bildhafte Vorstellungen: «Kerbtäler sind das Ergebnis fluviatiler Erosion, Muldentäler weisen auf eine glaziale Entstehung hin.» Die Talgenese erschöpft sich in den Vorstellungen der Schülerinnen und Schüler oftmals in aushobelnden Gletschern und tiefschürfenden Wildbächen. Die Oberrheinische Tief-

Martin Hasler, Dr., Geographisches Institut der Universität Bern, Hallerstraße 12, 3012 Bern

Urs Kaufmann, Vereinweg 1, 3012 Bern 


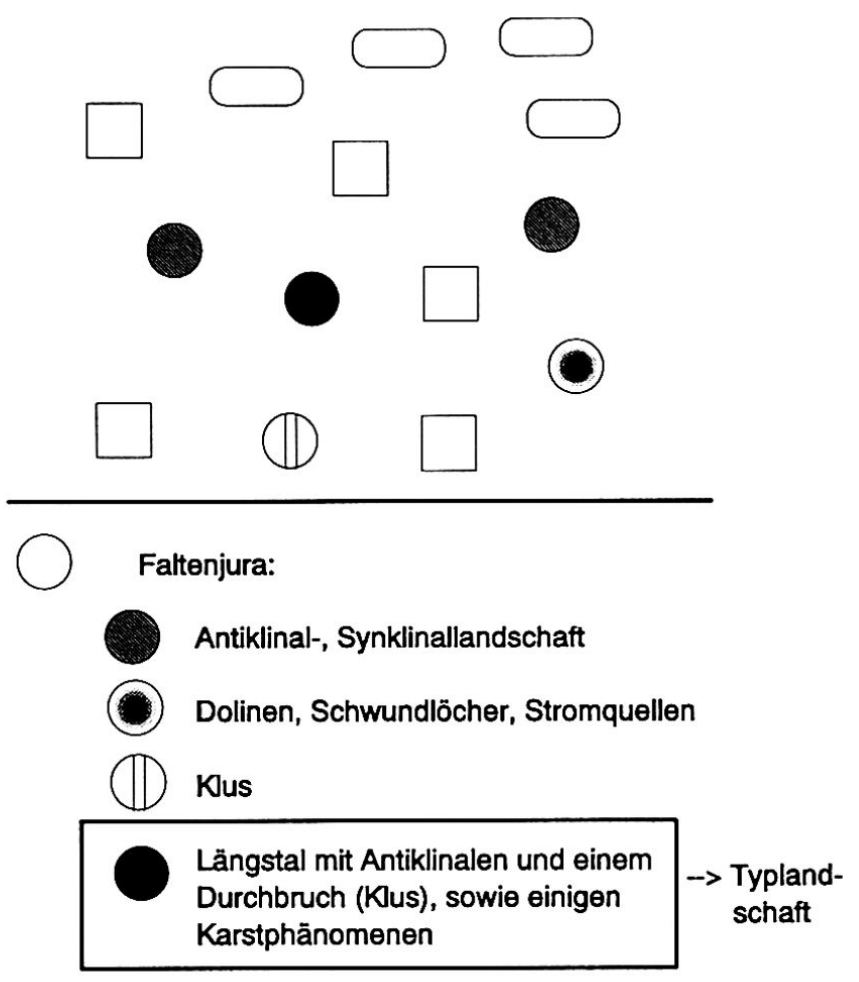

Tafeljura-Landschaften

Div. Oberflächenformen

Abb.1 Klassifikation von Typlandschaften am Beispiel der Geomorphologie des Faltenjuras.

ebene wird vor dem Hintergrund solcher Vorstellungen zu einem Glazialtal. Erübrigt sich der Geographieunterricht zudem im Aneinanderreihen typlandschaftlicher Betrachtungen, die möglichst noch alle nach dem gleichen Schema, von den Natur- zu den Kulturfaktoren (Oberflächenformen, Klima, Vegetation, Nutzungsgefüge), ablaufen, wird damit ein klischeehafter, stereotyper Geographieunterricht erzeugt, dem sowohl ein übergeordnetes Lernzielkonzept wie auch die didaktische Phantasie fehlen.

Die Lehrkraft wird sich immer wieder vom Spannungsfeld einer Landschaft gefordert sehen: Was läßt sich zu allgemeinen Erkenntnissen abstrahieren? Wo kann andererseits die Originalität und ästhetische Schönheit einer Landschaft dem Unterricht Spannung und Motivation verleihen? Gerade die kognitiv und arbeitsmethodisch orientierten Erkenntnisse und Fertigkeiten und die emotional ausgerichtete Spannung erzeugen jenen ganzheitlichen Ansatz, der den Bildungswert der Geographie charakterisiert.

\subsection{Von den Arbeitsblättern zum Unterricht}

Das Unterrichtswerk LANDSCHAFTEN DER SCHWEIZ (1993), das nun vollständig vorliegt, beruht auf der Idee, den Reichtum der Materialien, über die das Bundesamt für Landestopographie verfügt, dem Geographieunterricht vermehrt zugänglich zu machen. Getragen von der Lehrmittelkommission des Vereins Schweizerischer Geographielehrer (VSGg) unter Leitung von Peter Enzen, haben sich Geographielehrkräfte in einem Kurs der Weiterbildungszentrale (WBZ) auf das nun vorliegende Konzept geeinigt und die Arbeiten verteilt. Entstanden sind die folgenden Werke:

1. Die Arbeitsblätter, im Format A3 gefalzt ( $=4$ A4-Seiten), verfügen über Ausschnitte aus der Landeskarte, historische Karten, Bildmaterial, statistisches Material und über einen kurzen Kommentar, z. T. ergänzt durch Fragen und Aufgaben.

Man entschied sich für folgende Typlandschaften:
1. Goldau
9. Binntal
2. Rhonetal bei Leuk
10. Lötschental
3. Val de Travers
11. Monthey
4. Freiburg
12. Oberes Glattal
5. Cheseaux
13. Baden
6. Oerlikon
7. Zermatt
14. Zihlebene bei Cressier
15. Talkessel von Schwyz

8. Hochrheintal bei Basel

2. Der Kommentarband (LANDSCHAFTEN DER SCHWEIZ 1993, Band II) ist für die Hand der Lehrkraft gedacht und bietet eine knappe fachliche Vertiefung, weitere Arbeitsideen und Lösungsvorschläge an.

3. Die didaktischen Grundlagen zum vorliegenden Material liefert Band I der LANDSCHAFTEN DER SCHWEIZ (1993).

4. Auf einer Diskette ist zudem der EXCEL-Datensatz zu allen von den Typlandschaften tangierten Gemeinden abgespeichert. Statistiken lassen sich so auch problemlos selber nachführen.

5. Diareihen einiger bearbeiteter Typlandschaften werden in der Berner Schulwarte (Helvetiaplatz 2, 3005 Bern) ausgeliehen.

Das Gesamtwerk fand auch international Anerkennung und wurde 1994 an der Worlddidac mit dem Bronzeaward ausgezeichnet. Alle Materialien können bei der Geographica Bernensia in Bern bestellt werden.

\begin{tabular}{|llll|}
\hline \multicolumn{2}{l}{ Landschaften } & der Schweiz: & \\
Kommentar & S 6.1 & Didaktische Grundlagen (d oder f; 118 Seiten) & 20.- \\
Kommentar & S 6.2 & 15 kommentierte Arbeitsblätter (d/f; ca. 400 Seiten) & $60 .^{-}$ \\
Arbeitsblätter & Nr. 1-15: & 1.- pro Stück oder -.80 ab 25 Stück & \\
\hline Bezugsquelle: & Arbeitsgemeinschaft GEOGRAPHICA BERNENSIA, Hallerstraße 12, 3012 Bern & \\
\hline
\end{tabular}


fachwissenschaftliche Struktur

Problemstellung

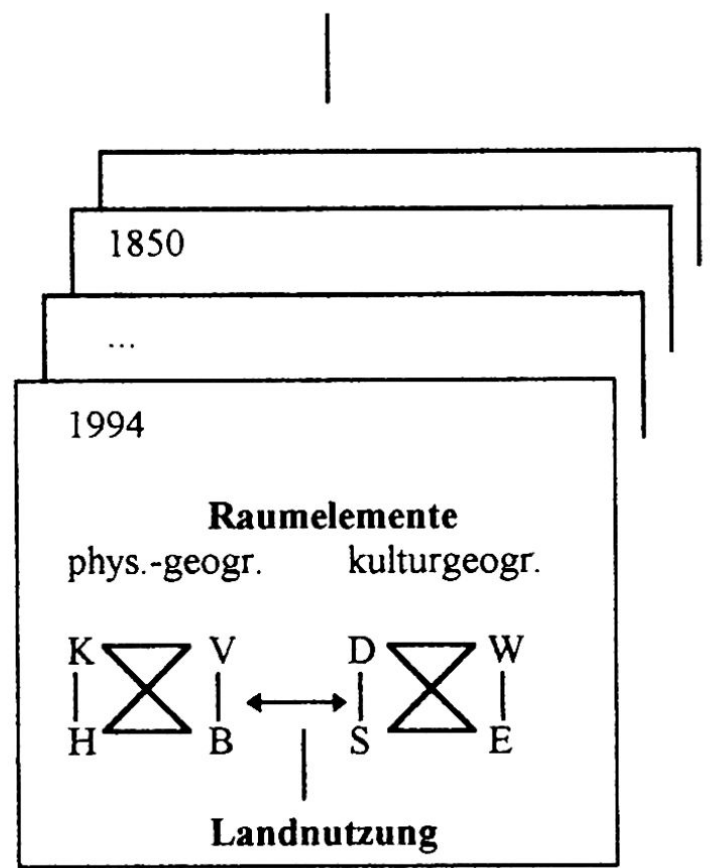
$\mathrm{K}$ : Klima
$\mathrm{V}$ : Vegetation
H: Hydrosphăre
B: Boden
D: Demographische Struktur
W: Wirtschaft
S: Gesellschaft
E: Ethisch-normatives System

\section{Lernprozesse}

Motivation

fragen lernen

Erarbeitung: 1. Raumelemente erfassen

2. Verknüpfung zu einem Raumgefuge

3. Landnutzung: Quantifizierung der Prozesse in Raum und Zeit

sehen lernen - Arbeitsmethodik

- Arbeitsformen (soziales Lernen)

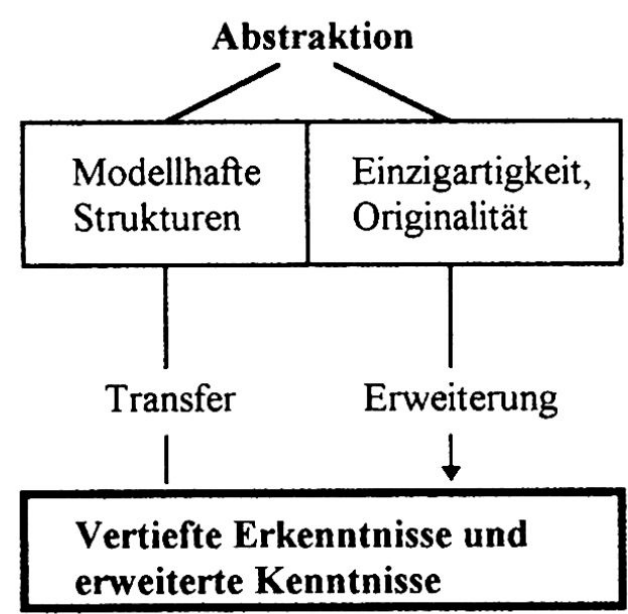

Abstrahieren: Modell und Wirklichkeit

Auswertung:

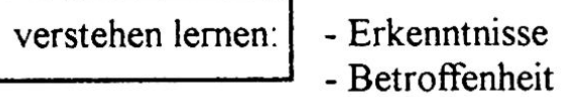

Synthese:

beurteilen lernen



Persönliches Verhalten überdenken 


\begin{tabular}{|c|c|c|c|c|}
\hline \multirow[t]{2}{*}{ Kategorien } & & \multicolumn{3}{|c|}{ Beitrag der Typlandschaften } \\
\hline & & bedeutungslos & bedingt & wichtig \\
\hline kognitiv & $\begin{array}{l}\text { - Analyse } \\
\text { - Synthese }\end{array}$ & $\begin{array}{l}\square \\
\square\end{array}$ & $\begin{array}{l}\square \\
\square\end{array}$ & 冈 \\
\hline affektiv & $\begin{array}{l}\text { - Betroffenheit } \\
\text { - eigenes Verhalten }\end{array}$ & $\begin{array}{l}\square \\
\square\end{array}$ & 田 & $\begin{array}{l}\text { 口 } \\
\square\end{array}$ \\
\hline instrumental & $\begin{array}{l}\text { - Umgang mit } \\
\text { Karte, Luftbild } \\
\text { - vergleichende } \\
\text { Arbeitsweise }\end{array}$ & $\begin{array}{l}\square \\
\square\end{array}$ & $\begin{array}{l}\square \\
\square\end{array}$ & $\begin{array}{l}\otimes \\
\otimes\end{array}$ \\
\hline sozial & $\begin{array}{l}\text { - Zusammenarbeit } \\
\text { - Enweiterung des } \\
\text { soziokulturellen } \\
\text { Horizontes }\end{array}$ & $\begin{array}{l}\square \\
\square\end{array}$ & $\boldsymbol{\otimes}$ & 口 \\
\hline ethisch & $\begin{array}{l}\text { - eigene Wertskala } \\
\text { - Toleranz }\end{array}$ & $\begin{array}{l}\square \\
\square\end{array}$ & 匈 & $\begin{array}{l}\square \\
\square\end{array}$ \\
\hline ästhetisch & $\begin{array}{l}\text { - Landschaft als } \\
\text { Erlebnis } \\
\text { - Persönliches } \\
\text { Erleben }\end{array}$ & $\begin{array}{l}\square \\
\square\end{array}$ & $\begin{array}{l}\square \\
\text { ब }\end{array}$ & $\begin{array}{l}凶 \\
\square\end{array}$ \\
\hline
\end{tabular}

Martm Havler, 1995

Abb.3 Eignungsmatrix der Arbeitsblätter "Typlandschaften" für den Geographieunterricht der Sekundarstufe II. Die Beurteilung kann unter Berücksichtigung spezieller Schulsituationen zu abweichender Einschätzung führen.

2 Arbeitsblatt Nr. 13, «Baden»: Beispiel einer didaktischen Umsetzung

Im folgenden soll am Beispiel Baden ein Arbeitsblatt näher vorgestellt und sollen die vielfältigen Einsatzmöglichkeiten in der Schule durch eine beispielhafte Unterrichtsskizze angedeutet werden.

\subsection{Voraussetzungen}

Das Arbeitsblatt Nr. 13, «Baden», eignet sich mit seiner großen Materialsammlung sehr gut dazu, die schulischen Einsatzmöglichkeiten aufzuzeigen. Folgende Quellen liegen vor:

- ein farbiges Schrägluftbild mit einem Überblick der Stadt Baden
- ein farbiger Kartenausschnitt 1:25000 von Baden 1843, 1904, 1988 (s. Abb. 4)

- der Stadtplan von Baden aus dem Jahr 1642 von Matthäus Merian (s. Abb. 4)

- ein ausführlicher Text über die Besiedlung und das Wachstum der Region Baden

- ein geologisches Blockbild durch die Klus von Baden

- Daten zur Bevölkerungsentwicklung der Region Baden

- Schüleraufgaben.

Für die Hand des Lehrers steht ferner ein 14seitiger Kommentar mit den Lösungen der Aufgaben, vertiefenden Zusatzinformationen sowie mehreren Abbildungen zur Verfügung (Band S 6.2).

\subsection{Unterrichtsskizze "Baden - von den Thermalquellen zum Dienstleistungszentrum"}

Am Fallbeispiel "Baden» kann exemplarisch die Entwicklung einer Siedlung vom Mittelalter bis heute untersucht werden. Die strategische Bedeutung der Limmatklus und die aufsteigenden Thermalwasser führten zu einer ersten Besiedlung in der Römerzeit, später zu einer mittelalterlichen Befestigung mit zugehörigem Dorf und schließlich mit dem Stadtrecht zur mittelalterlichen Stadt. Die Entwicklung Badens zu einer Industriestadt im 19. Jahrhundert und schließlich der Wandel zu einem Dienstleistungszentrum kann beispielhaft aufgezeigt werden. Diesen funktionalen Wandel haben in den letzten 200 Jahren fast alle Schweizer Städte durchlaufen, selten jedoch in solcher Deutlichkeit wie Baden. Wie ein roter Faden ziehen sich dabei die Standortfaktoren Badens durch die Entwicklungsgeschichte. Sie bilden die eigentlichen Eckpunkte, welche je nach technischer Entwicklung und sozio-kulturellem Umfeld entsprechend in Wert gesetzt werden. Die wichtigsten Phasen dieser Entwicklung zeigen Stettner \& Zweifel (AERNI et al. 1993, 170 , verändert) wie folgt:

\begin{tabular}{|lll|}
\hline Lagefaktor & ursprüngliche Bedeutung & heutige Bedeutung \\
\hline $\begin{array}{l}\text { Der geologisch tiefste Einschnitt } \\
\text { in den Untergrund: } \\
\text { die Thermalquellen. }\end{array}$ & $\begin{array}{l}\text { Das römische Baden «Aquae } \\
\text { Helveticae»... } \\
\text { (ca. 20 n. Chr. bis 400 n. Chr.) }\end{array}$ & $\begin{array}{l}\text {.. wurde zum Bäderquartier und } \\
\text { Kurzentrum. }\end{array}$ \\
\hline $\begin{array}{l}\text { Der Jurakalksteinsporn } \\
\text { und die Klusenge: }\end{array}$ & $\begin{array}{l}\text { Das mittelalterliche Baden im } \\
\text { Schutze der Festung bekam 1297 } \\
\text { das Stadtrecht und wurde 1798 } \\
\text { Hauptort des Kantons. }\end{array}$ & $\begin{array}{l}\text { Der Stadtkern wandelte sich zum } \\
\text { Kultur- und Dienstleistungszen- } \\
\text { trum mit Verwaltungsfunktion. }\end{array}$ \\
\hline $\begin{array}{l}\text { Die Schotterterrasse } \\
\text { im Innern der Klus: }\end{array}$ & $\begin{array}{l}\text { Das neuzeitliche Baden nach } \\
\text { de BBC-Grundung 1891 zog } \\
\text { Menschen und Arbeitsplätze an. }\end{array}$ & $\begin{array}{l}\text { Nebst dem Bahnhof und dem } \\
\text { Industrieareal entstanden die } \\
\text { Wohnquartiere. }\end{array}$ \\
\hline $\begin{array}{l}\text { Die anschließenden Terrassen- } \\
\text { fluren des Limmattales: }\end{array}$ & $\begin{array}{l}\text { An den Hängen anlehnende } \\
\text { Dörfer bildeten ein Arbeitskrafts- } \\
\text { und Baulandpotential. }\end{array}$ & $\begin{array}{l}\text { Sie bieten als Standorte der } \\
\text { Zulieferbetriebe und als zusätzli- } \\
\text { cher Wohnraum die Grundlage } \\
\text { der wirtschaftlichen Entfaltung } \\
\text { der Agglomeration. }\end{array}$ \\
& & \\
& &
\end{tabular}



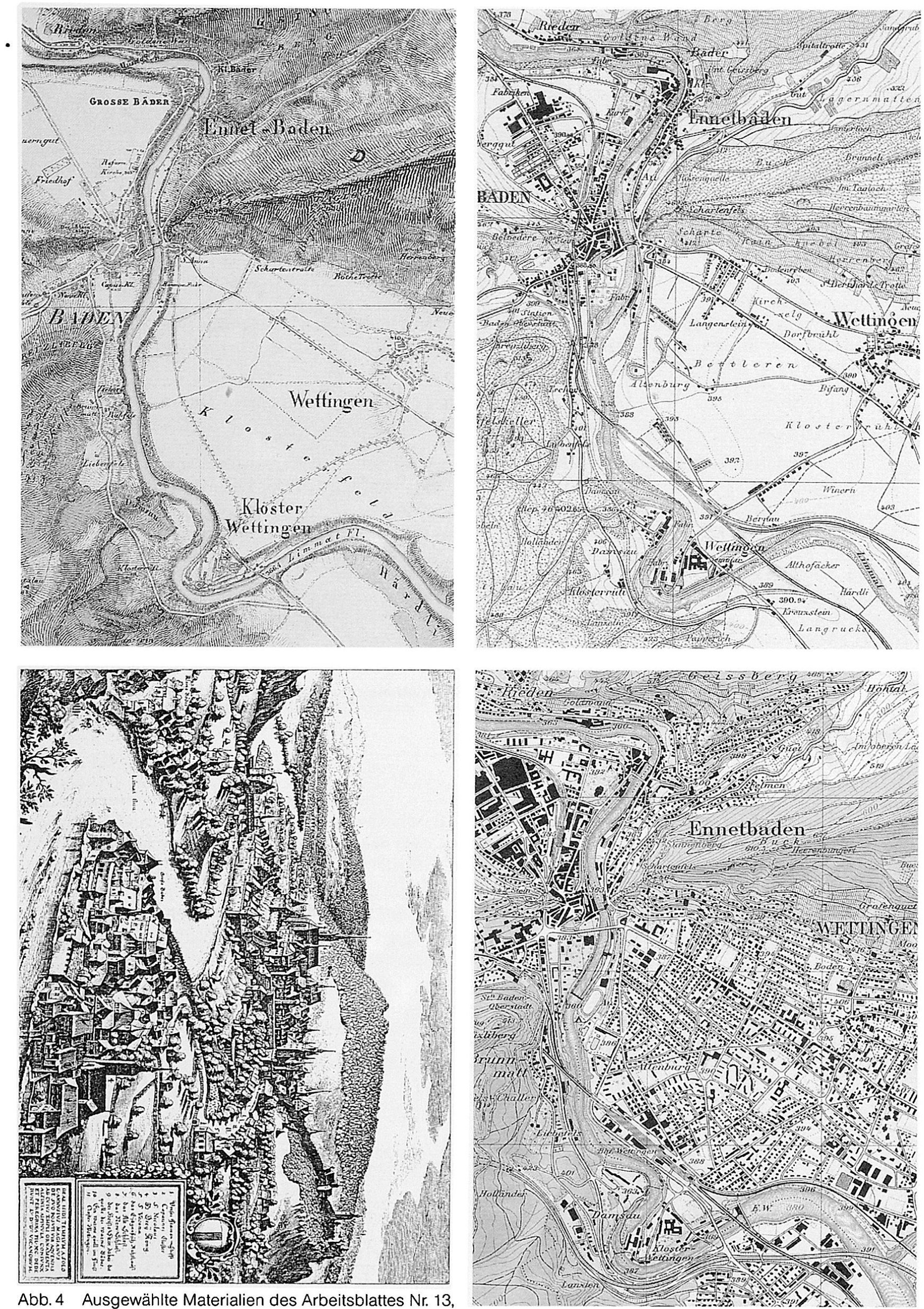

Abb. 4 Ausgewählte Materialien des Arbeitsblattes Nr. 13, "Baden" (verkleinert). 
Auf diese Überlegungen stützt sich die vorgeschlagene Unterrichtsskizze:

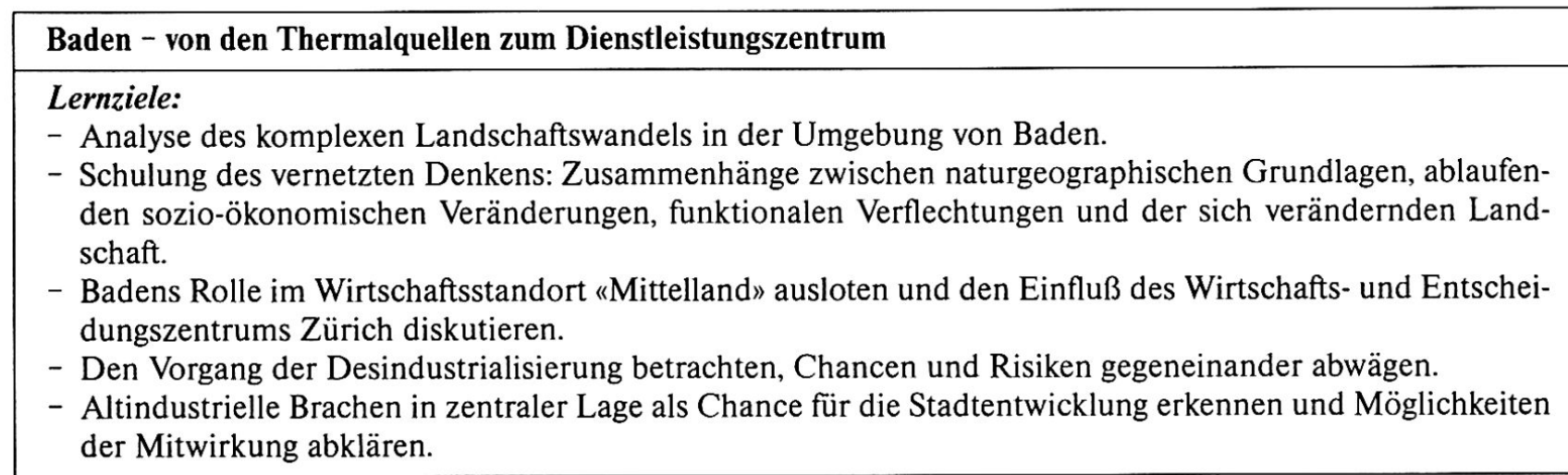

\begin{tabular}{|c|c|c|c|}
\hline Lektion & Ziele & Unterrichtsform & verwendete Quellen \\
\hline 1 & $\begin{array}{l}\text { Motivation: } \\
\text { Standortfaktoren bestimmen } \\
\text { die Siedlungslage: } \\
\text { - naturdeterministisch } \\
\text { - sozio-ökonomisch }\end{array}$ & $\begin{array}{l}\text { Kartenvergleich: Wichtigste } \\
\text { Entwicklungen und deren } \\
\text { Grundlagen diskutieren }\end{array}$ & $\begin{array}{l}\text { Kartenausschnitte Baden } \\
1843,1904,1988 \\
\text { LK 1:25000 Baden } \\
\text { Schülerkarte der Schweiz }\end{array}$ \\
\hline 2 und 3 & $\begin{array}{l}3 \text { wichtige Phasen } \\
\text { sozio-ökonomischer } \\
\text { Entwicklung Badens: } \\
\text { Unterschiedliche Bedeutung } \\
\text { der Standortfaktoren } \\
\text { beachten. }\end{array}$ & $\begin{array}{l}\text { Arbeitsteilige Gruppenarbeit: } \\
\text { - Baden im Mittelalter } \\
\text { - Baden um } 1900 \\
\text { - Baden heute } \\
\text { Referate der Gruppen }\end{array}$ & $\begin{array}{l}\text { Text des Arbeitsblattes } \\
\rightarrow \text { Michaeliskarte, Merianplan } \\
\rightarrow \text { Siegfriedkarte und Luftbild } \\
\rightarrow \text { Landeskarte und Luftbild } \\
\text { Bevölkerungsdaten }\end{array}$ \\
\hline 4 und 5 & $\begin{array}{l}\text { Detailanalyse } \\
\text { des heutigen Baden: } \\
\text { - Siedlungsentwicklung } \\
\text { - Verkehrsentwicklung } \\
\text { - Erholungsraum }\end{array}$ & $\begin{array}{l}\text { Kartierung der verschiedenen } \\
\text { Bebauungstypen nach der } \\
\text { Systematik von Grosjean } \\
\text { (AERNI et al. 1993: 83) } \\
\text { In Partnerarbeit aus den drei } \\
\text { Kartenausschnitten ergründen; } \\
\text { mit Schülertext überprüfen } \\
\text { Begriff Erholungsraum klären; } \\
\text { Luftbild- und Kartenanalyse }\end{array}$ & $\begin{array}{l}\text { Luftbild; evtl. zusätzlich Stadt- } \\
\text { plan, Lösungsvorschlag } \\
\text { (AERNI et al. 1993: 171/169) } \\
3 \text { Kartenausschnitte und Textteil } \\
\text { des Arbeitsblattes } \\
\text { LK 1988; Luftbild }\end{array}$ \\
\hline 6 und 7 & $\begin{array}{l}\text { Ein Blick in die Zukunft: } \\
\text { - Erholungsraum } \\
\text { - Verkehr } \\
\text { - Altindustrieareal }\end{array}$ & $\begin{array}{l}\text { Planungsideen } \\
\text { in Arbeitsgruppen: } \\
\text { Verbesserungs- und } \\
\text { Vernetzungsvorschläge } \\
\text { Möglichkeiten der Verkehrs- } \\
\text { führung und Parkplatzkonzept } \\
\text { Welche neuen Nutzungsformen } \\
\text { in welchen Bebauungstypen? } \\
\text { Vorstellen und Diskussion } \\
\text { der Lösungsvorschläge }\end{array}$ & $\begin{array}{l}\text { LK } 88 \text { und Luftbild } \\
\text { Zusätzlich Stadtplan } \\
\text { (AERNI et al. 1993: 171); } \\
\text { LK 1070 Baden } \\
\text { wie oben } \\
\text { Vergleich mit konkreten } \\
\text { Vorschlägen der Gemeinde }\end{array}$ \\
\hline
\end{tabular}


Es folgen nun Hinweise und Anregungen zu den einzelnen Abschnitten der Unterrichtseinheit:

\section{Lektion 1: \\ Die Standortfaktoren bestimmen die Siedlungslage}

Die Arbeit mit den drei Kartenausschnitten unterschiedlicher Zeit bietet an sich ausreichend Motivation, sich mit dem Raum Baden auseinanderzusetzen. Gerade durch den Einbezug der Michaeliskarte, welche mit ihrer Schraffentechnik wertvolle Akzente setzt, läßt sich nicht nur inhaltlich, sondern auch kartographisch mit einem fruchtbaren Vergleich der drei Zeitschritte einsetzen, welcher die Problematik der Region ganzheitlich zeigt. Diese erste Annäherung kann dann durch die Diskussion der Standortfaktoren vertieft werden.

Die naturbedingten Standortfaktoren lassen sich am besten aus der Michaeliskarte herauslesen, weil 1843 noch weite Teile Badens und der angrenzenden Gemeinden unverbaut sind. Dadurch ist das Relief besonders gut sichtbar. Allerdings ist der wichtigste Faktor, die Klus der Limmat durch die Lägernfalte des Juras, nur teilweise auf dem Ausschnitt zu erkennen. Daher empfiehlt sich der Einbezug der Landeskarte (LK, Blatt 1070, Baden).

Als wichtigste Landschaftselemente werden herausgearbeitet:

- der Lauf der Limmat

- die Klus mit ihren 2 Engnissen

- die Schotterterrassen innerhalb und außerhalb der Klus

- die Jurafalte mit ihren Südhängen

- die begrenzenden Molasseberge im Südwesten.

Diese Landschaftselemente am Übergang Jura-Mittelland bilden den Grundraster, worauf sich die sozio-ökonomischen Standortfaktoren abstützen und der die entsprechende Inwertsetzung begründet:

- Der Flußlauf als Transportweg, mit Brückenstandorten (Zolleinkünfte), als Energielieferant (Merianplan: Wasserrad im Handwerkerquartier, durch den Stadtbach angetrieben), in Schutzfunktion (für das Kloster Wettingen).

- Die Klus mit den Thermalquellen an der Überschiebungslinie Faltenjura/Tafeljura, wo an einer Querstörung $48^{\circ}$ warmes Wasser mit hohem Mineralgehalt aufsteigt, das schon von den Römern zu Heilzwecken genutzt wurde (vgl. tektonisches Blockbild des Arbeitsblattes).

Die Engnisse der Klus, die sich zur strategischen Kontrolle der Transportwege anbieten (Festung Stein, erstes Dorf im Schutz der Burg).

Das erhöhte Gefälle der Limmat im Klusbereich ermöglicht eine effiziente Energieproduktion (auf allen Kartenausschnitten ersichtlich).

- Die Schotterterrassen eignen sich hervorragend für alle möglichen Nutzungsformen: Landwirtschaft, Industrie, Dienstleistungen, Verkehr, Wohnraum.

- Die Südhänge der Jurafalte werden schon früh für der Rebbau genutzt (Michaeliskarte: «goldene Wand»).
Andererseits bietet die sonnenexponierte Hanglage auch Raum für privilegierte Wohnquartiere.

- Die bewaldeten Nordhänge der Molassehügel, welche den engeren Raum der Agglomeration Baden begrenzen.

- Der Einfluß der Agglomeration Zürich mit den daraus resultierenden Veränderungen der Pendlerströme, der Arbeitsplätze, der Besiedlung Badens; die Stellung Badens in bezug auf das schweizerische Hauptverkehrsnetz.

Nach dieser Vorarbeit setzt die gruppenweise Erarbeitung der sozio-ökonomischen Entwicklung Badens in drei Zeitschnitten ein.

\section{Lektionen 2 und 3:}

Drei wichtige Phasen in der Entwicklung

der Stadt und Region Baden

In diesen drei Zeitschnitten läßt sich schön der Wertewandel durch die technisch-industrielle Entwicklung und die dadurch veränderte Bedeutung der Standortfaktoren verfolgen. Aus den im Arbeitsblatt vorliegenden Quellen können folgende Themenkreise erschlossen werden:

- Baden im Mittelalter: Die Michaeliskarte zeigt ein ansprechendes Bild der Umgebung von Baden im Mittelalter, auch wenn sie aus der Zeit von 1843 stammt: Die Brückenübergänge, der Unterschied Stadt (Baden) Dorf (Wettingen), die Bedeutung der Bäder, die Besitzesverhältnisse (Klosterfeld), die Erwerbszweige (Landwirtschaft und Handel/Gewerbe/Verwaltung).

- Baden um 1900: Auf der Siegfriedkarte von 1904 zeigt sich Baden im Industriezeitalter. Zwei Eisenbahnlinien kreuzen sich im Bahnhof Wettingen und berühren Baden, die ursprüngliche Streckenführung der Spanischbrötlibahn (ohne Flußüberquerungen) bleibt sichtbar.

Die Bebauung wird anfänglich geprägt durch die Industriebauten der 1891 gegründeten BBC in der verkehrstechnisch gut erschlossenen Eisenbahnkurve nördlich von Baden. Weitere Industriebauten liegen auf der anderen Seite der Limmat im Dorfteil Rieden der Gemeinde Obersiggenthal.

Die neu entstandene Industrie führt zu einem Bevölkerungswachstum, welches sich in der Form von Arbeiterreihenhäusern zwischen dem Industriegelände und der Stadt Baden einerseits auf dem Klosterfeld in Wettingen andererseits auf der Siegfriedkarte erkennen läßt.

Neu entstanden sind auch das Kurhaus und der Kurpark im Bäderbereich. 


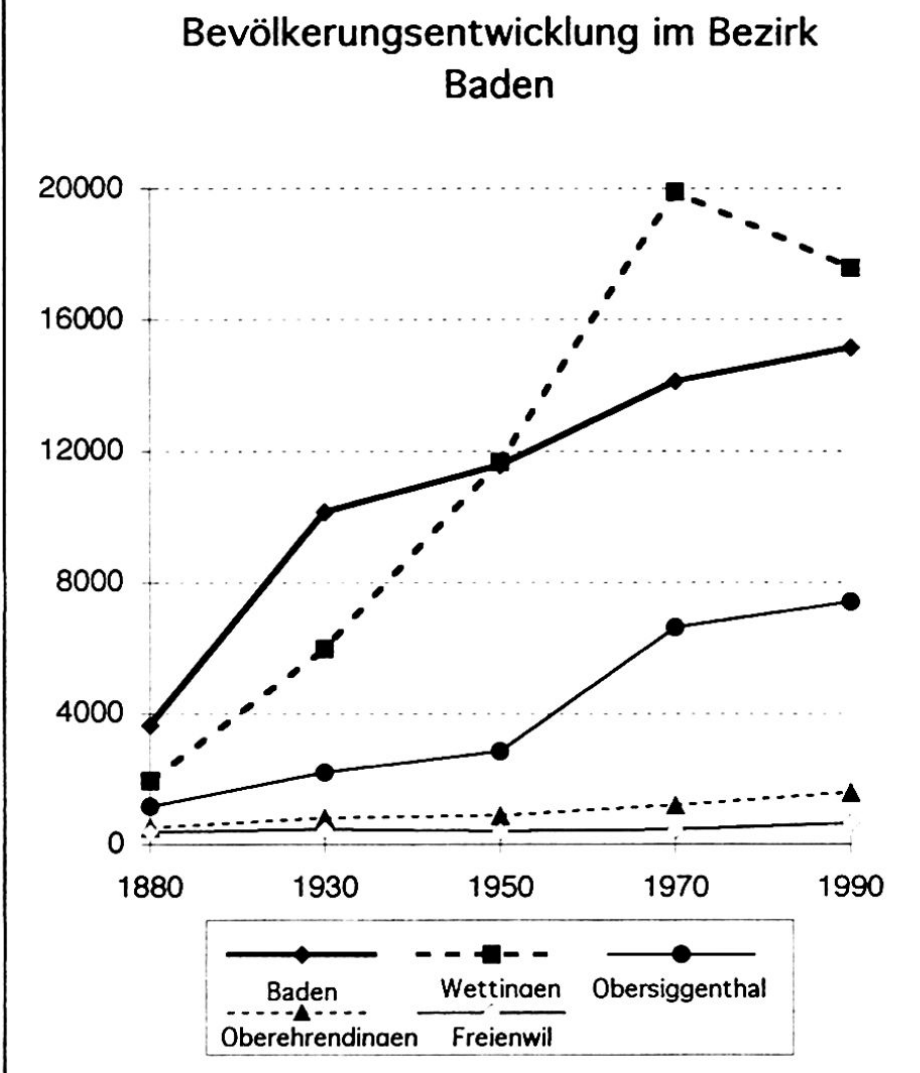

\section{Anteil der Betriebe im II. Sektor in \%}
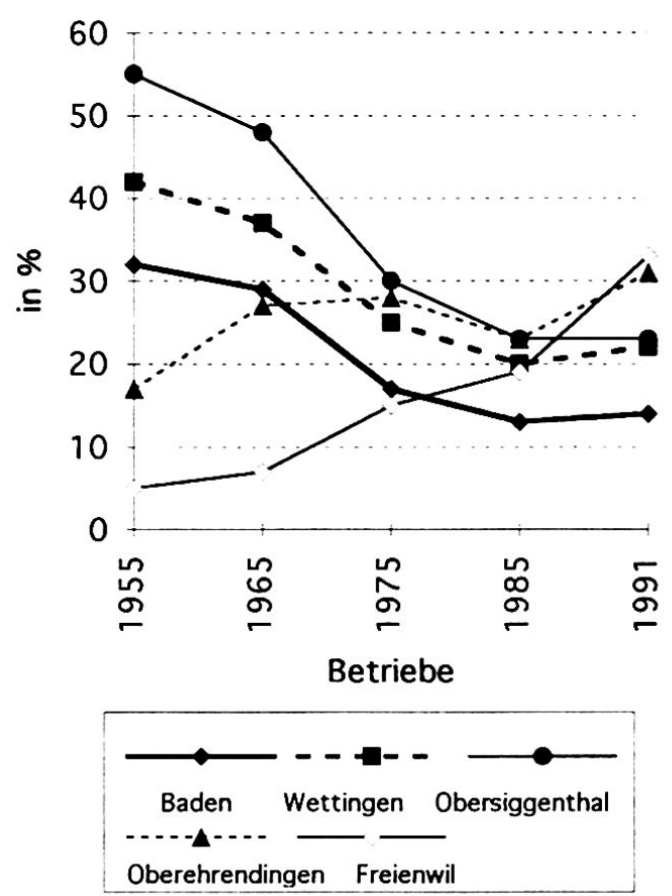

Abb.5 Bevölkerungsentwicklung und Anteil der im II. Sektor tätigen Firmen in ausgewählten Gemeinden der Agglomeration Baden.

Dank einer eigenen Signatur ist auf der Siegfriedkarte der Umfang des Rebbaus ersichtlich, welcher die nach wie vor vorhandene Bedeutung der Landwirtschaft sichtbar macht.

- Baden heute: Die Landeskarte von 1988 und das Luftbild geben ein gutes Bild vom Baden der Gegenwart. Die große Flächenbeanspruchung durch die Industrie täuscht jedoch: Längst hat diese in Baden selbst an Bedeutung verloren und ist weiter in die Peripherie abgewandert. Die große Persistenz der Gebäude zeigt diese Entwicklung nicht. Trotz der Desindustrialisierung steigt die Bevölkerung der Agglomeration Baden weiter an, die bereits mit derjenigen Zürichs nahezu zusammengewachsen ist (Raumplanungsbericht 1987: 10). Der Einfluß des Wirtschaftsschwerpunktes Zürich widerspiegelt sich in diesem Vorgang ebenso wie die allgemeine Tertiärisierung in der Schweiz. Mit Hilfe der Bevölkerungsstatistik auf dem Arbeitsblatt sowie den Daten zu den Anteilen des II. Sektors (aus dem entsprechenden Kommentar S 6.2: 173, ergänzt) ergeben sich folgende Verhältnisse auf einer ZentrumPeripherie-Achse (Baden, Wettingen, Obersiggenthal, Oberehrendingen, Freienwil):
Diese Entwicklung mit den entsprechenden Sättigungseffekten läßt sich teilweise auf der Karte nachvollziehen: Die begrenzten Raumverhältnisse der Stadt Baden ermöglichen kein bedeutendes Wachstum mehr. Deshalb überholt Wettingen mit der großen Baulandreserve des Klosterfeldes Baden bezüglich der Einwohnerzahl bereits in den 50er Jahren. Nachdem auch das Klosterfeld und ein Teil der Rebhänge vollständig überbaut sind, stagniert auch die Entwicklung von Wettingen, das Wachstum findet weiter außen in der Agglomeration statt.

Neue Industriebauten lassen sich in den Gemeinden Wettingen und Neuenhof erkennen (LK 88); die übrigen Außengemeinden sind auf dem Kartenausschnitt nicht erkennbar. Die Grafik zeigt aber deutlich die Peripherisierung der Industriebetriebe. Die ursprünglichen, durch Wasserkraft und Bahnanschluß bedingten Standorte wurden teilweise aufgegeben, die Wasserkraft dient heute der Stromerzeugung (mehrere Wehre).

Nach dieser gründlichen Studie der sozio-ökonomischen Entwicklung der Region Baden bietet es sich geradezu an, das heutige Baden vertieft zu analysieren. 
Lektionen 4 und 5:

Detailanalyse des heutigen Baden

Für die Detailanalyse bieten sich die Siedlungs- und Verkehrsentwicklung, eventuell zusätzlich die Erholungsfunktion an.

- Siedlungsentwicklung: Eine hervorragende Quelle ist das Luftbild, ergänzt durch den Ausschnitt der Landeskarte. Für Detailprobleme kann der Stadtplan beigezogen werden.

Als Untersuchungsmethode bietet sich ein Vorgehen nach Bebauungstypen an, welche auf dem Luftbild wiedererkannt werden können. Diese Methode wurde von Prof. G. Grosjean entwickelt und ist für Schülerinnen und Schüler angepaßt und erneuert worden (S 6.2: 83/84). Die Ziele liegen vorwiegend im formal-genetischen Erfassen der Bausubstanz. Die funktionalen $\mathrm{Zu}$ sammenhänge müssen interpretiert werden, da sie aus den Kartensignaturen nur ausnahmsweise hervorgehen. Durch eine Kartierung direkt auf das Luftbild oder auf ein aufgelegtes Kalkpapier kann eine übersichtliche Gliederung des Siedlungsbildes gelingen.

Folgende Bebauungstypen sind ersichtlich:

- Altstadtbebauung (gegliedert in Burgruine/Spornsiedlung, Verwaltungszentrum, Handwerkerquartier an der Limmat)

- Stadtkernbebauung mit Geschäften und Wohnungen, vorwiegend in Form von Blockrandbebauung

- Wohnquartiere in Form von Villenbebauung, Kleinhausbebauung, neuerer Quartierbebauung in Form von Wohnblöcken (in Zeilenbebauung) sowie verdichtetem Bauen

- Industriegebiet mit Fabriken

- öffentliche Gebäude (vor allem Schulen und Krankenheim)

- Grünflächen (Freiflächen, Wald, Landwirtschaft)

- Verkehrsentwicklung: Am deutlichsten erschließt sich die Verkehrsentwicklung aus dem Kartenvergleich 1843-1988. Stufenweise vermehren sich die Verkehrsträger und zeigen gleichzeitig die Reichweite und GröBe der vorhandenen Mobilität. Ein guter Ansatzpunkt ist eine Überlagerungskartierung auf Folie oder Kalk. Dabei kommt einerseits die große Persistenz gebauter Straßen und Schienenwege zum Vorschein, andererseits wird der enorme Ausbau seit den 60er Jahren deutlich. Der Schülertext zum Verkehr hilft bei der Karteninterpretation.

Ziel der Analyse sind die Veränderungen der Bedeutung der verschiedenen Verkehrsträger einerseits, die Abkoppelung Badens von den Hauptverkehrsachsen (N 1; Heitersberg) bei gleichzeitiger Zunahme der städtischen Verkehrsbelastung andererseits.

- Erholungsraum: Mit Hilfe einer Kartierung der Grünflächen kann der Erholungsraum erfaßt werden.

Natürlich gehören zur Erholungsfunktion nicht nur Grünflächen. Eine vertiefte Auseinandersetzung mit all dem, was die Menschen sich unter Erholung alles wünschen, führt erheblich weiter. Helfen kann eventuell eine Recherche mit dem (elektronischen) Telefonbuch oder ein Lehrausgang vor Ort.

\section{Lektionen 6 und 7:}

Ein Blick in die Zukunft:

Anstehende Probleme und Lösungsansätze

In der Schule kommt es heutzutage vermehrt darauf an, die Heranwachsenden auf die Möglichkeiten, den öffentlichen Raum mitzugestalten, aufmerksam zu machen. Dieser führt über eine politische Diskussion, welche nach einem Meinungsbildungs- und Mitspracheverfahren meist in einer Gemeindeabstimmung ihren Abschluß findet. Dieses (wenn auch bescheidene) Mitspracherecht muß genutzt werden.

Also gilt es, auch am Fallbeispiel Baden einen Blick in die Zukunft zu wagen. Dabei stehen die Themen Erholungsraum, Verkehr sowie die Neugestaltung des ABB-Areals (früher BBC) im Vordergrund.

- Erholungsraum: Es können planerische Verbesserungsvorschläge ausgedacht und eine ökologische Vernetzung der Grünzonen angestrebt werden. Nach einer Telefonbuchanalyse können auch Vorschläge im gastronomisch-, kulturell- und erlebnisorientierten Rahmen dazukommen.

- Verkehr: Trotz der Umfahrungen der Hauptverkehrsträger und des Stadttunnels erstickt Baden im Verkehr. Lösungen müßten in einer klaren Lenkung des regionalen Verkehrs um die Siedlung herum erfolgen. Hauptgewichte liegen im Zusammenspiel der verschiedenen Verkehrsmittel und einer geschickt konzipierten Parkraumbewirtschaftung.

- ABB-Areal: Die spannendste Herausforderung für Baden ist die Wiederbelebung dieses Altindustrieraumes. Eine große und zusammenhängende, zentrumsnahe Fläche wird frei und kann umgenutzt werden. Eine sorgfältige, ausgewogene Planung ist oberstes Gebot, handelt es sich doch bei diesen Dimensionen um ein Projekt von regionaler Bedeutung.

Sind die Schülerinnen und Schüler mit Baden nicht vertraut, empfiehlt sich eine Annäherung über die Daseinsgrundfunktionen (S 6.2: 85). Mit Hilfe dieser sieben Funktionen kann eine dem Standort entsprechende, vielseitige Lösung entworfen werden. Große Bedeutung ist dabei der Verkehrserschließung zu geben, damit sich die Verkehrsbelastung Badens nicht zusätzlich verschlimmert.

Diese Planungsversuche können mit den konkreten Vorhaben und Projekten der Gemeinde Baden verglichen werden. Die eigenen Projekte erfahren dadurch eine Wertung von außen. Ein Beispiel für die Planung des BBC-Areals liegt vor (S 6.2: 175). 


\section{Literatur}

ARBEITSBLÄTTER FÜR DEN GEOGRAPHIEUNTERRICHT, herausgegeben vom Bundesamt für Landestopographie und der Geographica Bernensia.

AERNI, K. (1993): Zur Auswahl und Bearbeitung von Typlandschaften. In: Aerni, K., Enzen, P., Kaufmann, U., 1993: Landschaften der Schweiz. Geographica Bernensia S 6, Band I. Bern.

GURTNER, M. (1993): Kartographie in der Schule - aus der Sicht eines Landestopographen. In: Aerni, K., Enzen, P., Kaufmann, U. (1993): Landschaften der Schweiz. Geographica Bernensia S 6, Band I. Bern.

HASLER, M. (1993): Typlandschaften im Unterricht - methodisch-didaktische Überlegungen. In: Aerni, K., Enzen, P., Kaufmann, U. (1993): Landschaften der Schweiz. Geographica Bernensia S 6, Band I. Bern.

JEANNERET, F. (1993): Typlandschaften und Geographie. In: Aerni, K., Enzen, P., Kaufmann, U. (1993): Landschaften der Schweiz. Geographica Bernensia S 6, Band I. Bern.
KAUFMANN, U. (1993): Mit Volldampf ins nächste Jahrtausend: Landschaftsveränderungen in der Schweiz. In: Aerni, K., Enzen, P., Kaufmann, U. (1993): Landschaften der Schweiz. Geographica Bernensia S 6, Band I. Bern.

KAUFMANN, U. (1993): Schulpraktische Hinweise zum Einsatz der "Arbeitsblätter für die Geographie. In: Aerni, K., Enzen, P., Kaufmann, U. (1993): Landschaften der Schweiz. Geographica Bernensia S 6, Band I. Bern.

LANDSCHAFTEN DER SCHWEIZ (1993), herausgegeben von K. Aerni, P. Enzen, U. Kaufmann. Band I: Didaktische Grundlagen. Analyse des Landschaftswandels anhand von Schweizer Typlandschaften und Entwicklungstypen. Band II: 15 kommentierte Arbeitsblätter für die Geographie des 9. bis 13. Schuljahres. Geographica Bernensia S 6. Bern.

STETTNER, N., ZWEIFEL, R. (1993): Kommentar zum Arbeitsblatt "Baden». In: Aerni, K., Enzen, P., Kaufmann, U. (1993) Landschaften der Schweiz. Geographica Bernensia S 6 , Band II. Bern.

SCHWEIZERISCHER BUNDESRAT (1987): Raumplanungsbericht. EDMZ 87.074. Bern.

THOENI, C. (1993): Kommentar zum Arbeitsblatt "Oerlikon". In: Aerni, K., Enzen, P., Kaufmann, U. (1993): Landschaften der Schweiz. Geographica Bernensia S 6, Band II. Bern. 